\title{
Better Engagement to Build Smarter, Resilient Communities
}

\author{
Alice Kesminas
}

Community resilience can be better achieved with geospatial information and technology, but only if we can determine what needs to be done for them to be accepted by communities. The challenge of trust, and concern about what else might be done with personal data beyond its initial purpose, need to be addressed to enable geospatial information to be used effectively to plan and manage resilient communities. We need to extend data anonymisation techniques to big data and understand what their limitations are when multiple data sources are linked.

\subsection{Introduction}

Geospatial information can improve our insights, understanding and management of events such as the COVID-19 pandemic, as well as the ongoing administration and planning of resilient communities and cities. Emerging technologies offer the opportunity to monitor our cities in real-time and gain an unprecedented level of location intelligence from a range of inputs - including mobile and wearable devices and other sensors that can identify an individual or household. Community reactions to tracing apps during the current COVID-19 pandemic have demonstrated that the public's concerns and expectations about technology and the privacy and security of their personal location data will determine how information can be used by decision makers. In this instance, technological capabilities have come second to the expectations of our communities. Community resilience can be better achieved with the help of geospatial information and technology, but only if we can determine what needs to be done for them to be accepted by communities.

Even with the potential for life-saving benefits, only around 7 million of Australia's 17 million adult population have downloaded the Australian Government's COVIDSafe app to date. "Like any health-care intervention, coronavirus apps need to conform to the highest standards of safety and efficacy. And yet, and there are no global standards" [1]. Lack of standards coupled with concerns about government use of data are possibly factors behind the current resistance to tracing apps. More than $60 \%$ of the population are "very concerned or concerned about their data being used by the Australian Government to make unfair decisions" [2]. Nonetheless there is support for data being made available to researchers (especially those in universities) and being used within government but there is much less support for multiple sources of data to be linked. The challenge of trust, and concern about what else might be done with personal data beyond its initial purpose, need to be addressed to enable geospatial information to be used effectively to plan and manage resilient communities. 


\subsection{Learning from Experience}

Open data is critical to smart growth and urbanisation. Sharing of sensor data, such as traffic flow, can allow multiple parties to develop insights that can improve day-to-day liveability and, via planning, the sustainability of our cites. While the benefits of sensors may be clear to councils, the installation of sensors to capture data has been known to cause issues. The City of Darwin found their "smart city" installation of sensors and cameras created issues within the community. In response to community expectations and privacy concerns, The City of Darwin committed to establishing a Privacy Framework to set the expectations and decision-making criteria for deployment of technology and data collection and management. They found it necessary to go beyond current privacy laws and help the community understand when their personal information is being collected and limit its use [3].

In retrospect, better community consultation could have reduced risks to the Victorian Smart Meter rollout. The technology was backed by a strong business case for better customer service, networks and safety but there was a need to clearly communicate benefits to individuals and address their concerns and lack of understanding about the new technology [4]. The sale of smart meter data to third parties to market alternative electricity plans and products to reduce energy consumption was pitched as a benefit by champions of the technology but is seen as a risk by some consumers. This mandated rollout was met with strong opposition and demonstrated that individual choice needs to be considered.

Engaging with the community from the outset was the key to success in the implementation of the Yackandandah mini grid [5]. By talking about the proposed new technology and listening to communities' concerns and vision, it was possible to co-develop a solution that would provide the network operator valuable insight into how to adapt to accommodate distributed energy resources and help the community to achieve their renewable energy vision. This is an example of what can be achieved when community, industry and government work together. We can learn from projects such as this by laying the foundation of trust and transparency for emerging geospatial technologies.

Community engagement can be costly, but there is also a cost when technology is not widely accepted in the community. The COVIDSafe app offered the promise of benefits to the community via reduced transmission, fewer lockdowns and less economic impact but still the download numbers were relatively small. The Australian Government's COVIDSafe app also suffered from public confusion about whether it even worked on some operating systems. If there was clearer communication, improved implementation and higher downloads, would we have captured critical transmissions earlier and avoided further costly lockdowns? What is the price we are paying, for not knowing where COVID-19 cases are sooner and having more robust contact tracing? What is the cost of not having community trust and acceptance of this technology?

\subsection{Extending Anonymisation to "Big" Geospatial Data}

With the increase in data being generated by the individual and analysed, maintaining privacy levels will be an increasingly complex issue. Data privacy has been identified as one of the biggest issues of the next decade [6]. There are several existing approaches to providing privacy for location data that includes sensitive or personal information such as address. These methods have been developed so that researchers and decision-makers can perform valuable spatial analysis, without the loss of personal privacy. Methods of anonymisation include, but are not limited to, aggregation and a range of masking, such as isomasks, where spatial analysis is performed in an offset location. As data and systems evolve, there is a need for new ways of ensuring privacy in increasingly complex systems. To achieve this, we need to extend data anonymisation techniques to big data and understand what their limitations are when multiple data sources are linked to support artificial intelligence. Once we understand privacy expectations, we can build the required solutions and legislation 
to support them. This means undertaking research now to ensure privacy can be guaranteed in increasingly complex systems, understanding de-anonymisation risks and determining who has the responsibility for protecting against them. It also means understanding the limitations of current acts as we transition to big data.

\subsection{Building Trust for Future Resilience}

Government, industry and communities can work together to: build trust and a mutual understanding of benefits technology offers to building resilient communities; provide expected levels of geospatial data security and privacy and; develop the standards and legislation that underpin these factors. Then we will be able to use smart technologies and geospatial data effectively build resilient communities.

The COVID-19 pandemic has provided valuable insight into the gap between technological possibilities and community acceptance of technology. As we ramp-up development digital twins as virtual replicas of our physical world and roll-out technology for smart cities technology, the reaction to tracing apps provides a timely reminder. Community engagement needs to underpin the process, not come as an afterthought. A range of issues have contributed to people's decision not to download tracing apps including bad press, rushed roll-out, platform incompatibilities and concerns about on selling of data. Similar issues in the future could be addressed with better community engagement as well as better design and implementation. If the community understands and accepts new geospatial technology, we can better use these technologies to build community resilience. We have an opportunity to learn from the reaction to tracing apps and achieve better outcomes in our future cities.

\section{References}

[1] Nature Editorial. Show evidence that apps for COVID-19 contact-tracing are secure and effective, 2020. URL https://go.nature.com/36TiT1w.

[2] N Biddle, B Edwards, M Gray, and S McEachern. Public attitudes towards data governance in Australia, 2018.

[3] City of Darwin. Switching On Darwin Privacy Framework, 2019. URL https://bit.ly/2GDoOgE.

[4] R. Bolt. The evolving engineer is the best engineer, ghd thoughtbook, 2020. URL https://bit.1y/2GErJpl.

[5] AusNet Services. Australia's first community mini grid launched in Yackandandah, 2017. URL https://bit. ly/3iMLYOC.

[6] P Thaine. Perfectly Privacy-Preserving AI, Towards Data Science, 2020. URL https://bit.1y/3nwna0Z. 
$\Longrightarrow$ Taylor \& Francis Taylor \& Francis Group

http://taylorandfrancis.com 